\title{
Observed Interstellar Extinction in the Ultraviolet ${ }^{1}$
}

\author{
T. P. STECHER
}

NASA Goddard Space Flight Center

Greenbelt, Maryland

$\mathrm{A}^{\mathrm{N}}$ observational program to measure stellar energy distributions completed. The instrumentation used was similar to that reported in reference 1. A necessary preliminary for discussion of this material is some knowledge of the extinction due to interstellar absorption. The trend of this extinction was first reported in 1964. (See ref. 2.) Additional observational material has since been obtained and is presented herein.

Five pairs of stars with similar Morgan-Keenan (MK) spectral classifications have been observed that are suitable for obtaining extinction: $\zeta$ Per and $\rho$ Leo; $\xi$ Per and $\zeta$ Pup; $o$ Per and $\beta$ Can Maj; $\sigma$ Sco and $\pi$ Sco; and $\delta$ Sco and $\tau$ Sco. The telemetry records were hand reduced by the author, each star was multiplied by the absolute calibration curve for the appropriate instrument, and the resulting flux curve was placed on a magnitude scale. Since interstellar extinction is obtained from the magnitude difference between two stars, the calibration factors completely cancel when both stars are observed by the same instrument. When the pair members are observed by different instruments, the consistency of the calibration from one instrument to another enters, but the absolute calibration does not. The instruments used for each reddening pair were calibrated at the same time, and comparison with stars observed by more than one instrument indicates that the error is negligible.

The extinction curve for each star pair has been normalized to a color excess $B-V=1$ by using colors reported in references 3 and 4 . For various observational reasons, the extinction curves are not com-

1 The contents of this paper were published previously in the Astrophys. J. (pub. by the Univ. of Chicago Press), vol. 142, 1965, p. 1683. 
plete for any star pairs. Therefore, the mean values $\Delta m$ and the standard deviation for the available points at each wavelength $\lambda$ are plotted in figure 1. A point-by-point computer reduction of all the star data is in progress and is expected to yield more pairs and greater accuracy for the existing pairs.

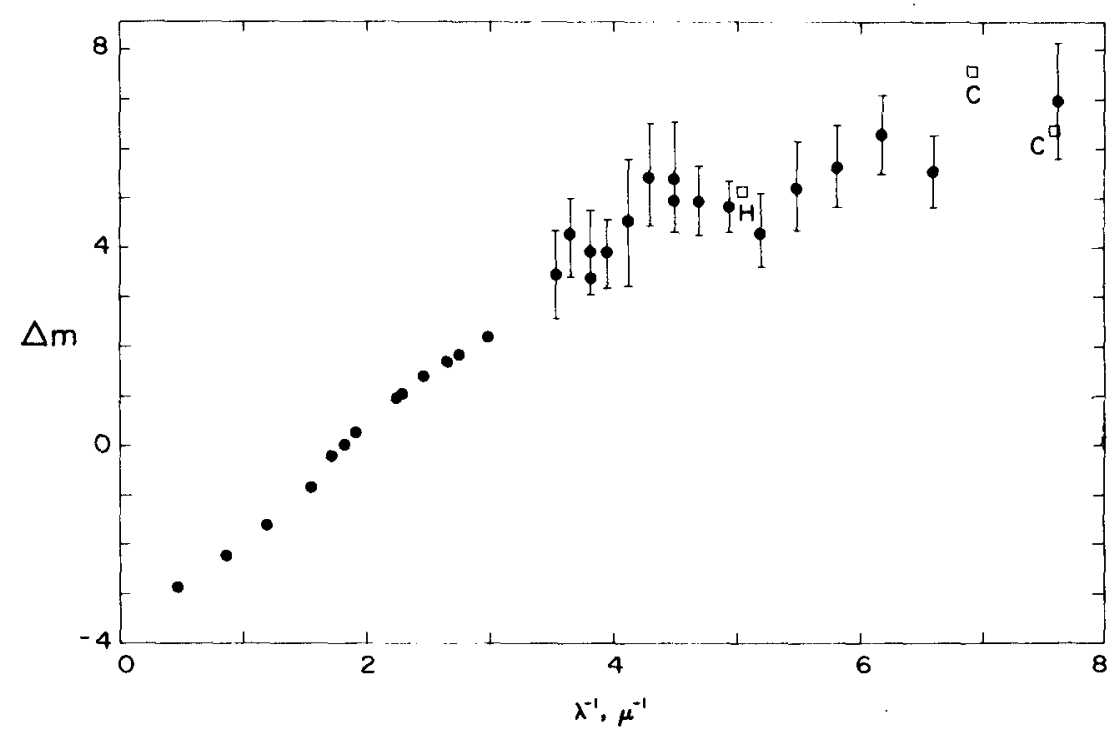

Figune 1.-Observed mean value for interstellar extinction. Solid circles: mean values for interstellar extinction and their mean errors for up to five pairs of stars as a function of inverse wavelength; solid circles without error bars: data from ref. 5; the square marked $H$ is from ref. 6; the squares marked $C$ are from ref. 7; all values are normalized with respect to $B-V=1$ and $V=0$.

In figure 1 , the mean extinction points are plotted, along with the mean extinction curve of reference 5 with $V=0$ for reference. An additional point was obtained from reference 6 , and two points were obtained from reference 7 . The separation between scatter that is intrinsic to extinction and observational scatter is difficult at this time, but for some stars the peak at $\lambda^{-1}=4.4$ microns $^{-1}$ appears to be considerably higher and drops off more rapidly before beginning to rise again. The results reported in reference 8 indicate that this variation is probably intrinsic. A possible explanation for the peak is suggested in the paper which follows.

\section{REFERENCES}

1. Stecher, T. P.; and Milligan, J. E.: Stellar Spectrophotometry from above the Atmosphere. Astron. J., vol. 136, 1962, p. 1. 
2. STEcher, T. P.: Ultraviolet Spectrophotometry of Early-Type Stars. Astrophys. J., vol. 69,1964 , p. 558.

3. Johnson, H. L.; and Bongman, J.: The Law of Interstellar Extinction. Bull. Astron. Inst. Netherlands, vol. 17, 1963, p. 115.

4. MacRae, D.: Observatory Handbook. Roy. Astron. Soc., Canada, 1961, p. 70.

5. Boggess, A.; and Borgman, J.: Interstellar Extinction in the Middle Ultraviolet. Astrophys. J., vol. 140, 1964, p. 1633.

6. Alexander, J. D.; Bowen, P. J.; Gross, M. J.; and Heddle, D. W. O.: Southern Hemisphere Observations of Ultra-violet Radiation from Celestial Objects I. Experimental Techniques and Rocket Payload Technology. Proc. Roy. Soc. (London), vol. A279, 1964, p. 510 .

7. ChubB, T. A.; and Byram, E. T.: Stellar Brightness Measurement at 1314 and $1427 \AA$ : Observation of the OI Twilight Glow. Astrophys. J., vol. 138, 1963, p. 617.

8. Johnson, H. L.: Interstellar Extinction in the Galaxy. Astrophys. J., vol. 141, 1965. p. 923. 